\title{
YBX1 promotes laryngeal squamous cell carcinoma progression via activating MAPK/ERK signaling as a target of miR-382-5p
}

\section{Wen Zeng}

Ganzhuo Cancer Hospital https://orcid.org/0000-0001-8238-8624

\section{Yiyun Pan}

Ganzhuo Cancer Hospital

Keqing Luo

Ganzhou Cancer Hospital

Keqiang Tian

Ganzhuo Cancer Hospital

Rong Li

Ganzhuo Cancer Hospital

Xiangmin Zhang ( $\nabla$ xmzhang019@163.com )

Ganzhuo Cancer Hospital https://orcid.org/0000-0003-2523-3938

\section{Research}

Keywords: Laryngeal cancer, Squamous cell carcinoma, microRNA, Y-box binding protein-1

Posted Date: April 3rd, 2021

DOl: https://doi.org/10.21203/rs.3.rs-379854/v1

License: (1) This work is licensed under a Creative Commons Attribution 4.0 International License.

Read Full License 


\section{Abstract}

Background. Y-box binding protein-1 (YBX1) influences the onset and progression of laryngeal squamous cell carcinoma (LSCC) remains unknown. The present study therefore sought to explore the mechanistic role of YBX1 in LSCC.

Methods.Analyses of the Gene Expression Omnibus (GEO) database and associated bioinformatics analyses revealed that YBX1 was upregulated in LSCC, and we further confirmed this result using primary LSCC patient samples. We additionally explored the impact of siRNA-mediated YBX1 knockdown on LSCC cell proliferation, migration, and invasion using CCK8, wound healing, and Transwell assays. We then conducted interrogated miRNA databases and conducted subsequent luciferase reporter assays to confirm that miR-382-5p binds to YBX1. Additional studies of the mechanisms downstream of this miR$382-5 p / Y B X 1$ axis focused on detecting the expression of mitogen-activated protein kinase (MAPK)/extracellular regulated kinase (ERK) signaling-related genes via qPCR and Western blotting.

Results.We detected significant upregulation of YBX1 in LSCC tumors that was significantly correlated with advanced TNM stage and poor patient prognosis. Knockdown of YBX1 markedly impaired the proliferative, invasive, and migratory activity of Tu212 cells in vitro. From a mechanistic perspective, miR382-5p was found to bind to the YBX1 3\&rsquo;-untranslated region and to thereby inhibit LSCC progression. We further confirmed that miR-382-5p negatively regulated YBX1 to inhibit proliferation via the MAPK/ ERK signaling axis in LSCC.

Conclusion. Together, our results indicated that YBX1 is an important promoter of LSCC progression, and that miR-382-5p can suppress YBX1 expression and inactivate MAPK/ERK signaling. These findings may thus highlight novel and promising prognostic and therapeutic targets in the context of LSCC.

\section{Introduction}

Laryngeal squamous cell carcinoma (LSCC) is a common epithelial tumor affecting the head and neck region[1], with roughly 13,430 newly diagnosed cases in 2016[2]. Roughly $60 \%$ of LSCC patients present with advanced disease that is often not amenable to treatment. Indeed, the overall survival (OS) rate for LSCC has declined slowly from $66-63 \%$ in recent decades, underscoring the severe morbidity and mortality of this cancer type[3]. The pathogenesis of LSCC is a complex multi-stage process that remains incompletely understood, and as such further studies of the mechanistic basis for these oncogenic processes have the potential to guide the diagnosis or treatment of this condition to improve patient prognosis.

Y-box binding protein-1 (YBX1) is a cold-shock protein that has previously been linked to dysregulated cell proliferation, drug resistance, and chromatin destabilization in the context of cancer[4]. Elevated YBX1 expression is associated with a poor prognosis in breast, prostate, lung, and ovarian cancers and in melanoma patients[5-8]. Higher YBX1 expression has also been reported that associated with more 
advances T stage, poorer tumor differentiation, and cervical or distance metastases in HNSCC patients[9]. The mechanisms whereby YBX1 modulates LSCC cell biology, however, remain to be elucidated.

MicroRNAs (miRNAs) are 20 nucleotide conserved non-coding RNAs that function as posttranscriptional regulators capable of binding to the 3 '-untranslated region (UTR) of target mRNAs to suppress their expression[10,11]. In the context of LSCC, many researchers have identified roles for these non-coding RNAs as regulators of cancer onset and progression [12]. MiR-382-5p is a miR-382 family member that is downregulated in many cancers, with such downregulation being linked to tumor progression [13]. In ovarian cancer, miR-382 has been shown to suppress tumor cell migratory and invasive activity via suppressing ROR1 expression and thereby impairing the epithelial-mesenchymal transition[14]. To date, however, no studies have explored miR-382-5p expression profiles or functional roles in the context of LSCC.

As such, in the present study, we explored the potential mechanisms whereby YBX1 and miR-382-5p may influence LSCC through in vitro and in vivo experiments. We found that YBX1 upregulation was common in LSCC tumor tissues, and that such upregulation was associated with enhanced LSCC tumor cell proliferative, migratory, and invasive activity. We further found that miR-382-5p directly targets YBX1 and suppresses its expression, thereby compromising LSCC tumor cell growth and activity. Restoring YBX1 expression to Tu212 cells overexpressing miR-382-5p was sufficient to enhance the proliferation, migration, and invasion of these cells. Together, these findings indicate that YBX1 plays an oncogenic role in LSCC and that it is negatively regulated by miR-382-5p, which might be a novel potential treatment target for LSCC

\section{Material And Methods}

\section{Cell lines, and reagents.}

The larynx carcinoma cell line TU212 cells were obtained fromGuangzhou Juyan Biological Technology (Guangzhou, Chin) and cultured in RPMI 1640 medium (Gibco; Grand Island, NY) with 10\% fetal bovine serum (FBS; Hyclone, Logan, UT), $100 \mathrm{U}$ penicillin and $100 \mathrm{mg} / \mathrm{ml}$ streptomycin (Gibco), hereafter referred to as standard media. The cells were kept at $37^{\circ} \mathrm{C}$ in a humidified atmosphere containing $5 \% \mathrm{CO} 2$.

\section{Tumor samples}

In total, 30 patients diagnosed with LSCC at the Ganzhou Tumor Hospital between 2014 and 2016 were enrolled in this study. Patients were an average of 63.6 years old (range: 28-79), and all patients underwent surgical treatment in our department. In addition, 15 normal paracancerous tissue segments ( $>1 \mathrm{~cm}$ from surgical margins) were collected to serve as control samples. Median patient follow-up was 27.1 months (Range: 2-85.6). Clinicopathological features of them are described in table 1. All patients provided written informed consent, and the Ethical Committee of Ganzhou Cancer Hospital approved this study(No.2017008).The association between gene expression and patient survival was assessed using Kaplan-Meier curves constructed in GraphPad Prism 8.0. 


\section{GEO data analysis}

GSE51985 and GSE59102 were obtained from the NCBI Gene Expression Omnibus (GEO) database (https://www.ncbi.nlm.nih.gov/gds). The download data format is MINIML,and the box plot is implemented by the R software package ggplot2. All data were downloaded from the public databases hence it was not required to obtain additional ethical approval for our study.

Table 1 Relationships Between YBX1 Expression in larygeal Tumor Tissues and Patients' Clinicopathological Features $(n=30)$

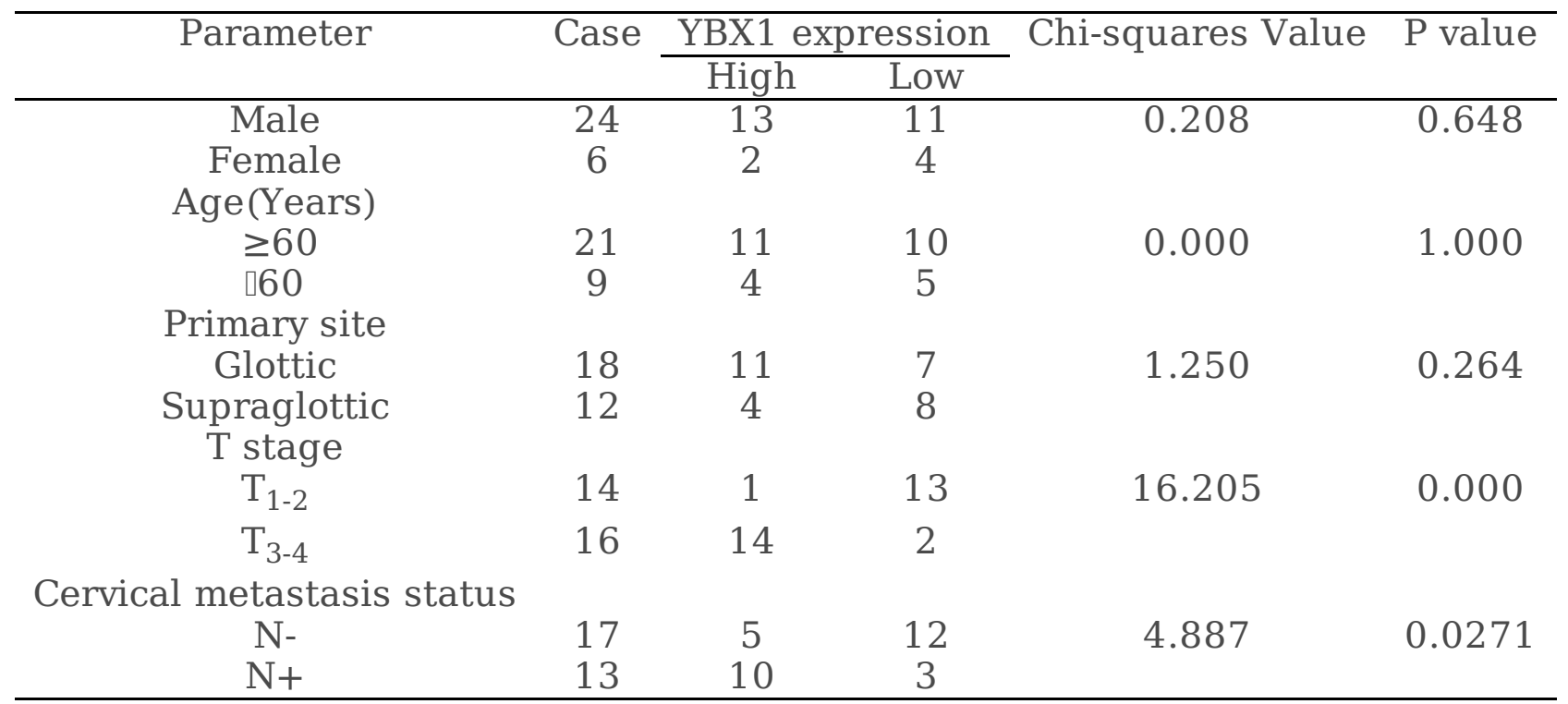

\section{Cell culture and transfection}

Human Tu212 LSCC cells were obtained from the Cell Bank of the Chinese Academy of Sciences (Shanghai, China) and were grown in DMEM (L110, Shanghai BasalMedia Technologies Co., LTD) containing $10 \%$ FBS and penicillin/streptomycin (15070063, Thermo, USA) in a $5 \% \mathrm{CO}_{2}$ incubator at $37^{\circ} \mathrm{C}$. Transfections were conducted by plating $5 \times 10^{4}$ cells/well of 6 -well plates and allowing them to grow until $60-70 \%$ confluent, at which time miR-382-5p (10,50, or $100 \mathrm{nmol} / \mathrm{L})$ was transfected into cells with Lipofectamine RNAiMAX (GenePharma, Shanghai, China), or plasmid DNA or siRNA duplexes (GenePharma) were transfected into cells using Lipofectamine 2000.

YBX1-homo-658 sense: 5-GGAGUUUGAUGUUGUUGAATT-3;

antisense:5-UUCAACAACAUCAAACUCCTT-3

YBX1-homo-906 sense: 5-GGUUCCCACCUUACUACAUTT-3;

antisense:5-AUGUAGUAAGGUGGGAACCTT-3

YBX1-homo-1007 sense: 5-GCAGGAGAACAAGGUAGACTT-3; 
antisense:5-GUCUACCUUGUUCUCCUGCTT-3;

Negative siRNA(NC) sense: 5-UUCUCCGAACGUGUCACGUTT-3;

antisense: 5-ACGUGACACGUUCGGAGAATT-3

\section{Migration and invasion assays}

For Transwell assays, $8 \mu \mathrm{m}$ inserts (BD Biosciences) that were or were not coated with $1 \mathrm{mg} / \mathrm{mL}$ Matrigel (Invitrogen) were seeded with $1 \times 10^{4}$ or $2 \times 10^{4}$ appropriate cells for invasion and migration assays, respectively. The lower chamber was then filled with DMEM containing $10 \% \mathrm{FBS}$, and cells were incubated for 24-48 h after which invasive/migratory cells were fixed, stained with crystal violet, and counted under $200 \times$ magnification.

\section{Cell viability assay}

Cells were transfected with miR-382-5p mimics, YBX1 overexpression plasmids, or YBX1-specific siRNA, after which a CCK8 kit (Beyotime, WI, USA) was used based on provided instructions to assess cell viability. Viability was assessed at $0,24,48,72$, and $96 \mathrm{~h}$ by assessing absorbance (OD) at $450 \mathrm{~nm}$ using a plate reader, with survival being calculated as follows: survival rate $=\left(O D_{\text {experimental }}-O D_{\text {blank }}\right) /\left(O D_{\text {control }}{ }^{-}\right.$ $\left.\mathrm{OD}_{\text {blank }}\right)$.

\section{Identification of YBX1-specific miRNAs}

To identify miRNAs that were predicted to bind to the YBX1 3'-UTR, we utilized four predictive tools including mirtarbase[15], starbase[16], miRwalk[17] and TargetScan[18].

\section{Luciferase reporter assay}

A Dual-Luciferase reporter assay was conducted based upon provided instructions (Promega, WI, USA). Briefly, HEK-293T cells were transduced with a lentivirus encoding miR-382-5p or a control construct and were then plated in 96 -well plates until $70 \%$ confluent. After an additional $12 \mathrm{~h}$, cells were co-transfected with $50 \mathrm{ng}$ of pMIR-YBX1-3'UTR-wt or pMIR-YBX1-3'UTR-mut and 10ng of pMIR-GLO (Gene Pharma). After a further $24 \mathrm{~h}$ incubation, the Dual-Luciferase Reporter Assay System was used to quantify firefly and Renilla luciferase activity.

\section{qRT-PCR}

TRIzol (Invitrogen, USA) was used to extract cellular RNA, after which a PrimeScript RT reagent kit (TaKaRa, Japan) was utilized to prepare cDNA, while a PrimeScript miRNA cDNA Synthesis Kit (TaKaRa) was instead used for miRNA analyses. SYBR Premix Ex Taq I was then used for qPCR analyses, and all primers used in this study are compiled in table 2. $\beta$-actin and U6 served as normalization controls for mRNA and miRNA analyses, respectively, with the $2-{ }^{-} \Delta \mathrm{Ct}$ approach being used to normalize gene expression. 
Table 2 Sequences used for qRT-PCR

\begin{tabular}{c|l}
\hline miR-382-5p & $\begin{array}{l}\text { F: ATCCGTGAAGTTGTTCGTGG } \\
\text { R:TATGGTTGTAGAGGACTCCTTGAC }\end{array}$ \\
\hline U6 & $\begin{array}{l}\text { F: CTCGCTTCGCRCAGCACA } \\
\text { R: AACGCTTCACGAATTTGCG }\end{array}$ \\
\hline YBX-1 & $\begin{array}{l}\text { F: GGTGGGTGTGTTGAAGAGAGAAGG } \\
\text { R: TCTAGGCTGTCTTTGGCGAGGAG }\end{array}$ \\
\hline K-Ras & $\begin{array}{l}\text { F: CATCTGCCTCCGAGTCCTGAATG } \\
\text { R: CACCATCTCTACCTCCACCTCCTC }\end{array}$ \\
\hline MEK & $\begin{array}{l}\text { F:CTTCCGTGGGTGTCGTGAATGG } \\
\text { R:TTCGCAGCAGATATAGGCAGGTTG }\end{array}$ \\
\hline p-MEK & $\begin{array}{l}\text { F: GCTCTTGTCTGGGAGCCAAC } \\
\text { R: GCACTTCCTCCCACTTCTGC }\end{array}$ \\
\hline ERK & $\begin{array}{l}\text { F: TCTCCTCTGTGTTGTCCTCCTTCC } \\
\text { R: GGCTGCCGCTCGACTTATGC }\end{array}$ \\
\hline p-ERK & $\begin{array}{l}\text { F: TCGCTCTGTCTCCAGGCTGAAG } \\
\text { R: CGGGAAGCTGAGGAAGAAGAATCG }\end{array}$ \\
\hline$\beta$-actin & $\begin{array}{l}\text { F: CCTGTACGCCAACACAGTGC } \\
\text { R: ATACTCCTGCTTGCTGATCC }\end{array}$ \\
\hline
\end{tabular}

\section{Western blotting}

Protein was extracted using RIPA lysis buffer, after which $50-100 \mu \mathrm{g}$ of protein per sample was separated via SDS-PAGE and transferred onto PVDF membranes (Roche, IN, USA). Membranes were then blocked using 5\% non-fat milk and incubated with appropriate primary antibodies: mouse anti-human YBX1 (1:1000, Abcam); rat anti-human k-RAS (1:3000, Abcam); mouse anti-human p-MEK (1:2000, Santa Cruz); mouse anti-human p-ERK (1:1000, Santa Cruz); mouse anti-human MEK (1:3000, Santa Cruz); mouse anti-human ERK (1:1500, Santa Cruz); mouse anti-human actin (1:3000, Sigma Aldrich). Enhanced chemiluminescence reagents (Thermo Scientific, MA, USA) were then used to detect protein bands on prepared blots.

\section{Immunohistochemistry (IHC)}

Paraffin-embedded tissue sections were deparaffinized using xylene, after which they were rehydrated with an ethanol gradient. Endogenous peroxidase activity was then blocked using $3 \% \mathrm{H}_{2} \mathrm{O}_{2}$, after which samples were heated in a microwave to facilitate antigen retrieval. Next, $5 \%$ BSA was used to block nonspecific antigen binding for 1 hour at $37^{\circ} \mathrm{C}$, after which sections were incubated with primary antiYBX1 (1:250, Abcam, USA) overnight at $4^{\circ} \mathrm{C}$. Sections were then incubated for $1 \mathrm{~h}$ with secondary antibodies at $37^{\circ} \mathrm{C}$, after which diaminobenzidine was used to detect antibody binding and sections were counterstained using hematoxylin. An Olympus light microscope was then used to capture representative images of tissue sections.

\section{Wound healing assay}

Wound healing assays were conducted to evaluate cellular migration. Briefly, cells were plated until 80$85 \%$ confluent, at which time a sterile micropipette tip was used to scratch the monolayer. PBS was then 
used to wash away damaged cells, after which DMEM containing $10 \%$ FBS was added and cells were incubated for $24 \mathrm{~h}$. The wound site was then imaged via phase-contrast microscope (Zeiss, Gottingen, Germany), with Image-Pro Plus v4.5.1 being used to quantify wound closure.

\section{Tumor xenograft model}

Tu212 cells that had been transfected with mir-382-5p with or without YBX1 overexpression were subcutaneously injected into the flank of 4-6 week-old female BALB/c nude mice $\left(1 \times 10^{7}\right.$ cells in $200 \mu \mathrm{l}$ PBS). Following a 28-day experimental period, tumor length and width ( $L$ and $W$, respectively) and weight values were assessed, and tumor volumes were calculated using the formula: volume $=1 / 2 \mathrm{LW}^{2}$. The Ganzhou Cancer Hospital Committee approved all animal studies.

\section{Statistical analysis}

Data are means \pm S.D. from three independent experiments, and were analyzed using SPSS v22.0 (IBM, USA). Data were compared using Student's t-tests, with Kaplan-Meier curves being used for survival analyses. $\mathrm{P}<0.05$ was the significance threshold.

\section{Results}

\section{YBX1 upregulation correlates with disease progression and poor LSCC patient prognosis}

To understand key genes associated with LSCC progression, we evaluated gene expression profiles associated with this cancer type in the publically available GSE59102 and GSE51985 dataset. We specifically focused on genes that were upregulated in LSCC as they may represent viable therapeutic targets or prognostic biomarkers in this disease type. Of these, we selected YBX1 as a gene that was highly upregulated in LSCC tumor tissues (Fig. 1a). We then validated these previously published results by measuring YBX1 expression in 30 LSCC patient tumor tissue samples and 15 control tissues via IHC and Western blotting. These analyses confirmed that YBX1 was significantly overexpressed in LSCC tumors relative to healthy tissues (Fig. 1b-c). We further found that YBX1 expression levels were significantly higher in patients with tumors of a more advanced TNM stage ( $t=17.63, P<0.001$, Fig. $1 d)$. Additional prognostic analyses prognostic analysis also revealed that higher $\mathrm{YBX} 1$ expression levels were correlated with decreased overall survival (OS) in LSCC patients ( $95 \% 1.130$ to $8.585, P=0.031$, Fig. 1 e). Together, these data indicated that YBX1 is upregulated in LSCC and is associated with tumor progression.

\section{YBX1 promotes LSCC tumor cell migratory and proliferative activity}

To understand whether YBX1 plays important roles in LSCC, we next conducted loss-of-function assays wherein we transfected Tu212 cells with siRNA constructs specifically targeting the YBX1 splice junction. Knockdown efficiency was confirmed via qPCR, with the YBX1-1007 and YBX1-658 siRNA constructs significantly reducing YBX1 levels in transfected cells (Fig.2a). Tu212 cells transfected with YBX1-1007 
were then analyzed in CCK8 assays, which demonstrated that YBX1 knockdown markedly suppressed LLC cell proliferation at all tested time points (48h, $p<0.01 ; 72 \mathrm{~h}$ and $96 \mathrm{~h}, \mathrm{p}<0.001$, Fig. $2 \mathrm{~b}$ ). In addition, transwell assays revealed that YBX1 knockdown impaired LSCC cell migratory and invasive activity relative to control cells (Fig.2c). Together, these findings indicate that YBX1 promotes metastasis in Tu212 cells.

\section{YBX1 is a miR-382-5p target in LSCC cells}

We next evaluated the regulatory mechanisms governing YBX1 expression in LSCC cells. Using the miRtarbase, miRWalk, starbase, and TargetScan databases, we identified three miRNAs (miR-379-5p, miR382-5p, and miR-137) with the potential to regulate YBX1 expression (Fig3a). We then elected to focus specifically on the role of miR-382-5p in this oncogenic context. To that end, we constructed luciferase reporters bearing WT and mutant versions of this putative miRNA binding site in the YBX1 3'-UTR (Fig3b). We confirmed that YBX1 knockdown was associated with the upregulation of miR-382-5p, whereas overexpressing miR-382-5p markedly suppressed YBX1 expression in Tu212 cells (Fig3c). We then transfected cells with a range of miR-382-5p concentrations and found that YBX1 expression was reduced at higher miRNA concentrations (Fig3d). Luciferase reporter assays further confirmed that the transfection of Tu212 cells bearing the WT but not the MUT YBX reporter plasmid was significantly reduced following miR-382-5p transfection (Fig3e).

\section{miR-382-5p suppresses YBX1 and thereby inhibits LSCC tumor growth}

We next conducted additional functional assays aimed at exploring the function of miR-382-5p-mediated YBX1 in the context of LSCC. To that end, we transfected Tu212 ${ }^{\text {miR-382-5p }}$ cells with a YBX1 overexpression vector (Tu212 ${ }^{\mathrm{miR}-330-5 p+Y B X 1}$ ). We found that overexpressing this miRNA significantly decreased the migratory and invasive ability of Tu212 cells, as expected (Fig.4a-c). Importantly, rescue experiments confirmed that overexpressing YBX1 in these cells restored their proliferative and migratory activity (Fig.4a, b). Using an in vivo subcutaneous tumor implantation model, we further found that tumors derived from Tu212 ${ }^{\text {miR-382-5p+YBX1 }}$ cells were significantly larger than tumors derived from Tu212 ${ }^{\text {miR-382-5p }}$ cells (Fig.4c). Together, these loss- and gain-of-function results confirm that miR-382-5p suppresses YBX1 expression and thereby impairs the growth of LSCC tumors.

\section{miR-382-5p inhibits YBX1 to inhibit LSCC growth via MAPK/ERK signaling}

Lastly, we explored the underlying signaling mechanisms whereby miR-382-5p-mediated repression of YBX1 may enhance LSCC tumor growth. As YBX1 is a known MAPK-dependent regulator of cellular proliferation and gene expression[19], we sought to specifically evaluate the role of MAPK signaling in the present experimental context. We found that YBX1 knockdown was associated with the inhibition of K-ras and MEK expression in Tu212 tumor cells (Fig 5a,b). We then evaluated the ability of miR-382-5p to indirectly modulate MEK/ERK signaling via YBX1. Through these analyses, we found that overexpressing miR-382-5p significantly impaired YBX1, K-Ras, and MEK levels in Tu212 cells, whereas overexpression of 
YBX1 was sufficient to restore the expression of these three signaling components in Tu212 ${ }^{\text {miR-382-5p }}$ cells(Fig5c,d). Together, these findings provide evidence that miR-382-5p can module MEK/ERK signaling via suppressing YBX1 expression and thereby altering MEK and ERK phosphorylation. Overall, we were therefore able to conclude that miR-382-5p inhibits LSCC tumor progression via suppressing YBX1 expression and thereby inactivating MEK/ERK signaling.

\section{Discussion}

LSCC patient death is often the result of tumor metastasis, which is a complex multi-stage process that is modulated by the expression of specific miRNAs and mRNAs. Herein, we found that YBX1 expression was elevated in patients with LSCC, and that such upregulation was associated with unfavorable clinicopathological findings and poor patient prognosis. Additional in vitro analyses revealed that YBX1 was able to modulate MAPK/ERK signaling and to thereby promote LSCC cell proliferative, migratory, and invasive activity, whereas miR-382-5p negatively regulated such activity. Overall, these findings suggested that miR-382-5p/YBX1 is a key signaling regulator in LSCC and may thus represent a novel clinical target for the treatment of this deadly cancer. To the best of our knowledge, no prior studies have demonstrated a role for the miR-382-5p/YBX1 axis in the regulation of LSCC progression.

YBX1 regulates many nucleic acid-related intracellular activities including DNA repair, pre-mRNA transcription and splicing, mRNA packaging, translation, and mRNA stability [20]. YBX1 functions as a transcription factor that can control differentiation, proliferation, drug resistance, and stress responses in many tumor types[21]. From a mechanistic perspective, YBX-1 silencing suppresses MMP1 and $\beta$-catenin expression in breast cancer cells, thereby disrupting their invasive activity. Herein, we explored the upstream mechanisms regulating YBX1 expression in LSCC, and we determined that elevated YBX1 expression was correlated with poor prognostic indicators including T and TNM stage. Additionally, survival analyses suggested that YBX1 expression levels are associated with decreased OS in LSCC patients. Together, these data indicate that YBX1 thus plays an oncogenic role in the context of LSCC development and progression.

Many studies have demonstrated that miRNAs are important regulators of key pathological processes linked with LSCC onset and progression, governing tumor cell proliferation and metastatic processes[22]. Using appropriate predictive databases, we ultimately determined that miR-382-5p targets YBX1 $[15,16]$. MiR-382-5p is generated from the $5^{2}$-terminus of the miR-382 pre-miRNA, and has previously been shown to be dysregulated and associated with progression in many cancer types[23]. For example, Du et al[24] determined that miR-382-5p is commonly downregulated in the context of liver cancer associated with hepatitis $B$ infections and that such low expression is often associated with tumor metastasis. In breast cancer, miR-382-5p additionally targets the RERG/Ras/ERK pathway to promote oncogenesis, allowing it to serve as a diagnostic or prognostic biomarker in this cancer type [25]. Prior studies, however, have not clarified the role of miR-382-5p in LSCC. Our findings provide clear evidence that miR-382-5p-mediated regulation of YBX1 inhibits LSCC progression. As such, miR-382-5p may be a viable biomarker for the detection and treatment of LSCC. 
We additionally studied the mechanisms whereby YBX1 may promote tumor progression in the present study. In prior reports, the RRM2/TK1/TYMS axis has been found to regulate nucleotide metabolism and to govern the proliferation and senescence of tumor cells[26]. YBX1 induces the proliferation of cells via promoting GSK3B/Cyclin D1/Cyclin E1 pathway activation in pancreatic ductal adenocarcinoma [27], and drives renal cell carcinoma metastasis through the G3BP1-SPP1-NF-KB signaling pathway[28]. Furthermore, YBX-1 has been shown to modulate Raf/MEK/ERK signaling so as to promote the progression of prostate cancer[29]. Herein, we determined that miR-382-5p targets YBX1 and thereby promotes the upregulation of $\mathrm{p}-\mathrm{MEK}$ and $\mathrm{p}$-ERK in LSCC, while negatively impacting k-RAS, MEK, and ERK levels. As such, miR-382-5p/YBX1 may inhibit ERK activation by suppressing RAS/MEK/ERK in LSCC cells, consistent with prior findings in the context of prostate cancer[29]. MAPK/ERK signaling pathway activity can be targeted with drugs including apatinib and anlotinib[30, 31]. As such, our results highlight a potentially novel approach that can guide future efforts to treat LSCC.

\section{Conclusion}

In conclusion, we identified YBX1 as a prognostic factor associated with the progression of LSCC. We additionally found that miR-382-5p directly targets YBX1 and thereby modulates MAPK/ERK signaling so as to inhibit LSCC progression. These findings thus suggest that targeting the miR-382-5p/YBX1 axis may be a viable approach to treating LSCC.

\section{Declarations}

\section{Acknowledgments}

None

\section{Competing interest}

The authors report no conflicts of interest in this work.

\section{Authors'contributions}

All authors contributed to the study conception and design. Xiangmin Zhang and Rong Li: conceptualization and funding acquisition. Keqing Luo, Keqiang Tian: resources and investigation and data curation.Junhui Xie: review and editing. Wen Zeng, Yiyun Pan: formal analysis and software and writing-original draft. All authors read and approved the final manuscript.

\section{Availability of data and materials}

Datasets are available on request from the corresponding author on reasonable request. The raw data and all related documents supporting the conclusions of this manuscript will be made available by the authors, without undue reservation, to any qualified researcher. 


\section{Ethics approval and consent to participate}

This retrospective chart review study involving human participants was in accordance with the ethical standards of the institutional and national research committee and with the 1964 Helsinki Declaration and its later amendments or comparable ethical standards. This study received the Ganzhou cancer hospital Ethical Committee approval (No.2017008).

\section{Funding}

This work was supported by the Natural Science Foundation of Jiangxi Province (Grant numbers.20181BAB205037), and Ganzhou Tumor Hospital Department Guidance Plan Project(Grant numbers. GZ2018ZSF363)

\section{Consent for publication}

Not applicable

\section{Competing interest}

The authors report no conflicts of interest in this work.

\section{References}

1. Cadranel J, Canellas A, Matton L, Darrason M, Parrot A, Naccache JM, et al. Pulmonary complications of immune checkpoint inhibitors in patients with nonsmall cell lung cancer. Eur Respir Rev. 2019;28:190058. https://doi.org/10.1183/16000617.0058-2019.

2. Siegel RL, Miller KD, Jemal A. Cancer statistics, 2016. CA Cancer J Clin. 2016;66:7-30. https://doi.org/10.3322/caac.21332.

3. Wang J, Wu Y, Gao W, Li F, Bo Y, Zhu M, et al. Identification and characterization of CD133(+)CD44(+) cancer stem cells from human laryngeal squamous cell carcinoma cell lines. J Cancer. 2017;8:497506. https://doi.org/10.7150/jca.17444.

4. Kosnopfel C, Sinnberg T, Schittek B. Y-box binding protein 1-a prognostic marker and target in tumour therapy. Eur J Cell Biol. 2014;93:61-70. https://doi.org/10.1016/j.ejcb.2013.11.007.

5. Cui Y, Li F, Xie Q, Zhao S, Guo T, Guo P, et al. YBX1 mediates autophagy by targeting p110 $\beta$ and decreasing the sensitivity to cisplatin in NSCLC. Cell Death Dis. 2020;11:476. https://doi.org/10.1038/s41419-020-2555-4.

6. Murugesan SN, Yadav BS, Maurya PK, Chaudhary A, Singh S, Mani A. Interaction network analysis of YBX1 for identification of therapeutic targets in adenocarcinomas. J Biosci. 2019;44:27.

7. Lim JP, Shyamasundar S, Gunaratne J, Scully OJ, Matsumoto K, Bay BH. YBX1 gene silencing inhibits migratory and invasive potential via CORO1C in breast cancer in vitro. BMC Cancer. 2017;17:201. https://doi.org/10.1186/s12885-017-3187-7. 
8. Kosnopfel C, Sinnberg T, Sauer B, Busch C, Niessner H, Schmitt A, et al. YB-1 Expression and Phosphorylation Regulate Tumorigenicity and Invasiveness in Melanoma by Influencing EMT. Mol Cancer Res. 2018; 16:1149-60. https://doi.org/10.1158/1541-7786.MCR-17-0528.

9. Zhao X, Zhang W, Ji W. YB-1 promotes laryngeal squamous cell carcinoma progression by inducing miR-155 expression via c-Myb. Future Oncol. 2018;14:1579-89. https://doi.org/10.2217/fon-20180058.

10. Ali Syeda Z, Langden SSS, Munkhzul C, Lee M, Song SJ. Regulatory Mechanism of MicroRNA Expression in Cancer. Int J Mol Sci. 2020;21. https://doi.org/10.3390/ijms21051723.

11. Kelly TJ, Brümmer A, Hooshdaran N, Tariveranmoshabad M, Zamudio JR. Temporal Control of the TGF- $\beta$ Signaling Network by Mouse ESC MicroRNA Targets of Different Affinities. Cell Rep. 2019;29:2702-17. https://doi.org/10.1016/j.celrep.2019.10.109.

12. Lin XJ, Liu H, Li P, Wang HF, Yang AK, Di JM, et al. miR-936 Suppresses Cell Proliferation, Invasion, and Drug Resistance of Laryngeal Squamous Cell Carcinoma and Targets GPR78. Front Oncol. 2020;10:60. https://doi.org/10.3389/fonc.2020.00060.

13. Du J, Bai F, Zhao P, Li X, Li X, Gao L, et al. Hepatitis B core protein promotes liver cancer metastasis through miR-382-5p/DLC-1 axis. Biochim Biophys Acta Mol Cell Res. 2018; 1865:1-11. https://doi.org/10.1016/j.bbamcr.2017.09.020.

14. Tan H, He Q, Gong G, Wang Y, Li J, Wang J, et al. miR-382 inhibits migration and invasion by targeting ROR1 through regulating EMT in ovarian cancer. Int J Oncol. 2016;48:181-90. https://doi.org/10.3892/ijo.2015.3241.

15. Kozomara A, Birgaoanu M, Griffiths-Jones S. miRBase: from microRNA sequences to function. Nucleic Acids Res. 2019;47:D155-62. https://doi.org/10.1093/nar/gky1141.

16. Li JH, Liu S, Zhou H, Qu LH, Yang JH. starBase v2.0: decoding miRNA-ceRNA, miRNA-ncRNA and protein-RNA interaction networks from large-scale CLIP-Seq data. Nucleic Acids Res. 2014;42:D92-7. https://doi.org/10.1093/nar/gkt1248.

17. Sticht C, De La Torre C, Parveen A, Gretz N. miRWalk: An online resource for prediction of microRNA binding sites. PloS one. 2018;13:e0206239. https://doi.org/10.1371/journal.pone.0206239.

18. Agarwal V, Bell GW, Nam JW, Bartel DP. Predicting effective microRNA target sites in mammalian mRNAs. Elife. 2015;4.https://doi.org/10.7554/eLife.05005.

19. Schäfer R, Sers C. RAS oncogene-mediated deregulation of the transcriptome: from molecular signature to function. Adv Enzyme Regul. 2011;51:126-36. https://doi.org/10.1016/j.advenzreg.2010.11.005.

20. Lyabin DN, Eliseeva IA, Ovchinnikov LP. YB-1 protein: functions and regulation. Wiley Interdiscip Rev RNA. 2014;5:95-110. https://doi.org/10.1002/wrna.1200.

21. Kuwano M, Shibata T, Watari K, Ono M. Oncogenic Y-box binding protein-1 as an effective therapeutic target in drug-resistant cancer. Cancer Sci. 2019;110:1536-43. https://doi.org/10.1111/cas.14006. 


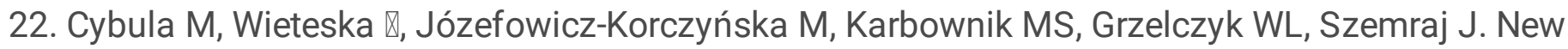
miRNA expression abnormalities in laryngeal squamous cell carcinoma. Cancer Biomark. 2016;16:559-68. https://doi.org/10.3233/cbm-160598.

23. Liu D, Zhong L, Yuan Z, Yao J, Zhong P, Liu J, et al. miR-382-5p modulates the ATRA-induced differentiation of acute promyelocytic leukemia by targeting tumor suppressor PTEN. Cell Signal. 2019;54:1-9. https://doi.org/10.1016/j.cellsig.2018.11.012.

24. Du J, Bai F, Zhao P, Li X, Li X, Gao L, et al. Hepatitis B core protein promotes liver cancer metastasis through miR-382-5p/DLC-1 axis. Biochim Biophys Acta Mol Cell Res. 2018;1865:1-11. https://doi.org/10.1016/j.bbamcr.2017.09.020.

25. Ho JY, Hsu RJ, Liu JM, Chen SC, Liao GS, Gao HW, et al. MicroRNA-382-5p aggravates breast cancer progression by regulating the RERG/Ras/ERK signaling axis. Oncotarget. 2017;8:22443-59. https://doi.org/10.18632/oncotarget.12338.

26. Kloetgen A, Duggimpudi S, Schuschel K. YBX1 Indirectly Targets Heterochromatin-Repressed Inflammatory Response-Related Apoptosis Genes through Regulating CBX5 mRNA. Int J Mol Cell. 2020;21:4453. https://doi.org/10.3390/ijms21124453.

27. Liu Z, Li Y, Li X, Zhao J, Wu S, Wu H, et al. Overexpression of YBX1 Promotes Pancreatic Ductal Adenocarcinoma Growth via the GSK3B/Cyclin D1/Cyclin E1 Pathway. Mol Ther Oncol. 2020;17:2130. https://doi.org/10.1016/j.omto.2020.03.006.

28. Wang Y, Su J, Wang Y, Fu D, Ideozu JE, Geng H, et al. The interaction of YBX1 with G3BP1 promotes renal cell carcinoma cell metastasis via YBX1/G3BP1-SPP1- NF-KB signaling axis. J Exp Clin Cancer Res. 2019;38:386. https://doi.org/10.1186/s13046-019-1347-0.

29. Imada K, Shiota M, Kohashi K, Kuroiwa K, Song Y, Sugimoto M, et al. Mutual regulation between Raf/MEK/ERK signaling and Y-box-binding protein-1 promotes prostate cancer progression. Clin Cancer Res. 2013;19:4638-50. https://doi.org/10.1158/1078-0432.ccr-12-3705.

30. Liu M, Wang X, Li H, Xu L, Jing L, Jiang P, et al. The effect of apatinib combined with chemotherapy or targeted therapy on non-small cell lung cancer in vitro and vivo. Thorac Cancer. 2019;10:1868-78. https://doi.org/10.1111/1759-7714.13162.

31. Hu H, Liu Y, Tan S, Xie XX, He J, Luo F, et al. Anlotinib Exerts Anti-Cancer Effects on KRAS-Mutated Lung Cancer Cell Through Suppressing the MEK/ERK Pathway. Cancer Manag Res. 2020;12:357987. https://doi.org/10.2147/cmar.s243660.

\section{Figures}


A

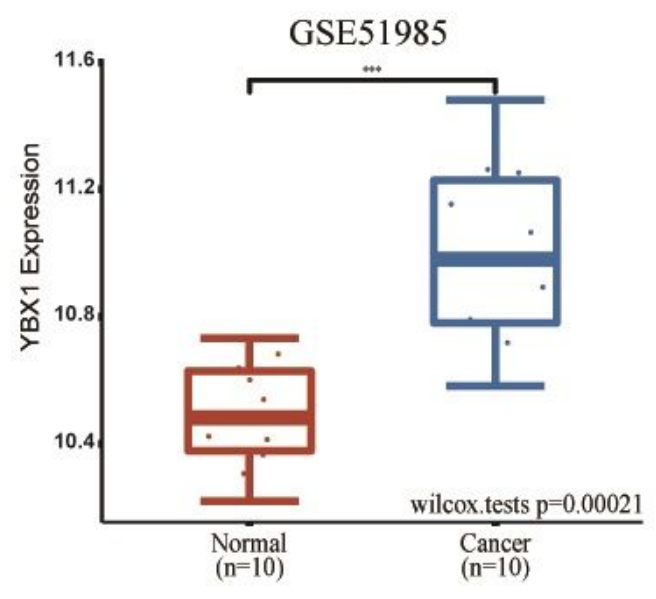

B

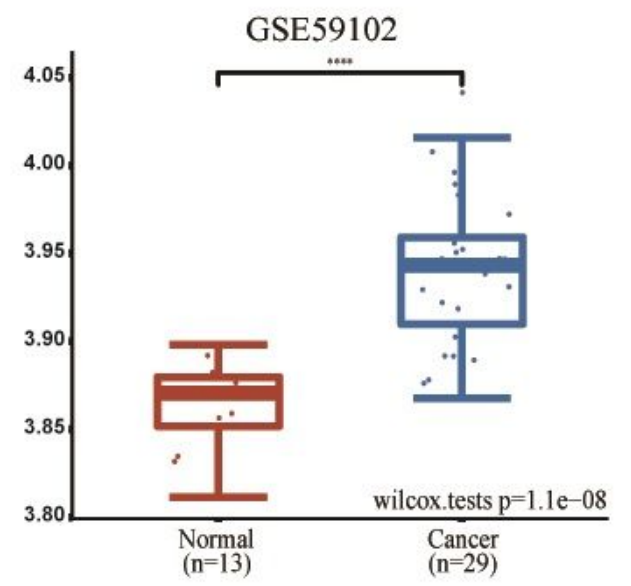

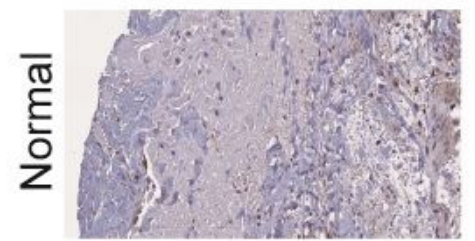

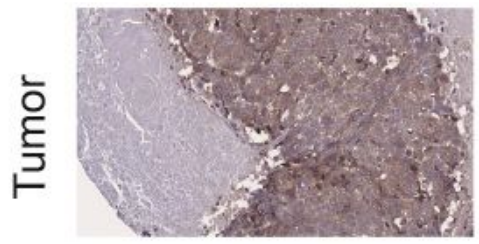

C

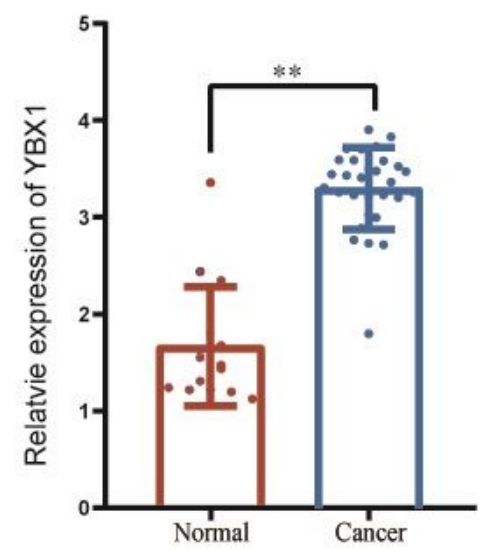

D

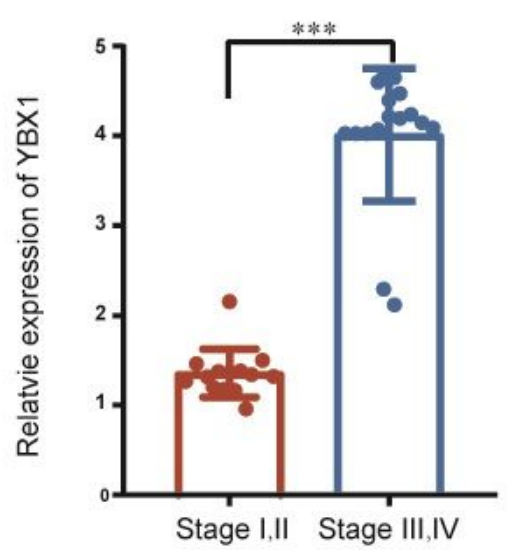

$E$

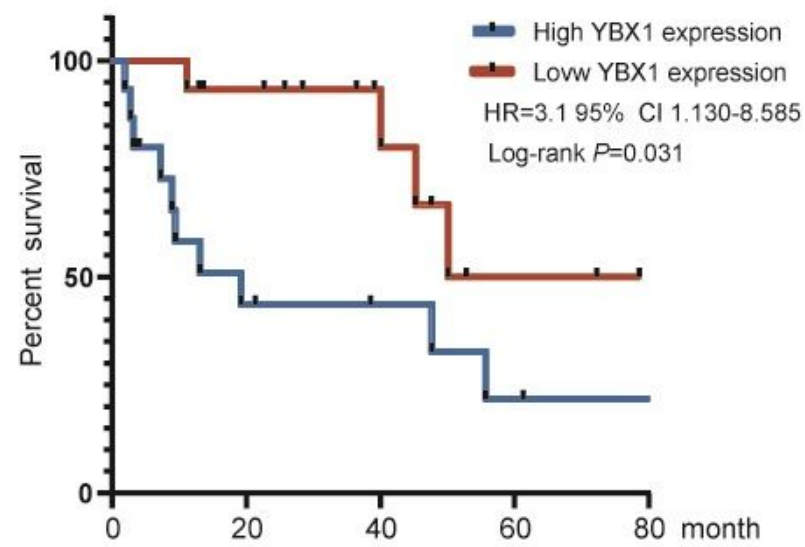

\section{Figure 1}

YBX1 expression is increased and correlated with poor prognosis in laryngeal squamous cell carcinoma. a Different expression of YBX1 in LSCC cancer relative to normal tissue controls, with genes expressed at low and high levels being shown in red and blur, respectively (GSE59102 and GSE51985). b YBX1 protein levels in LSCC tumors and control tissues were evaluated via IHC. c YBX1 expression was quantified via Western blotting in 30 LSCC tumor samples and 15 normal control tissue samples.d Relative YBX1 expression levels in early-stage $(I, I, n=14)$ and advanced-stage $(I I I, I V, n=16)$ LSCC tissues were measured. e The association between laryngeal YBX1 expression and OS in LSCC patients was assessed. Data are means \pm SD. ${ }^{\star *} P<0.01,{ }^{*} * * P<0.001$; Student's t-test. 
A

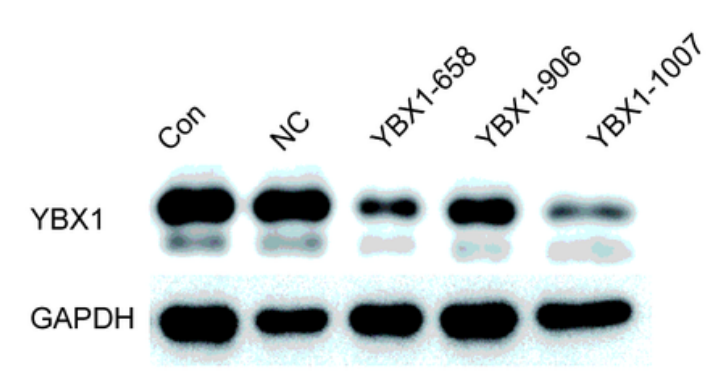

$\mathrm{C}$

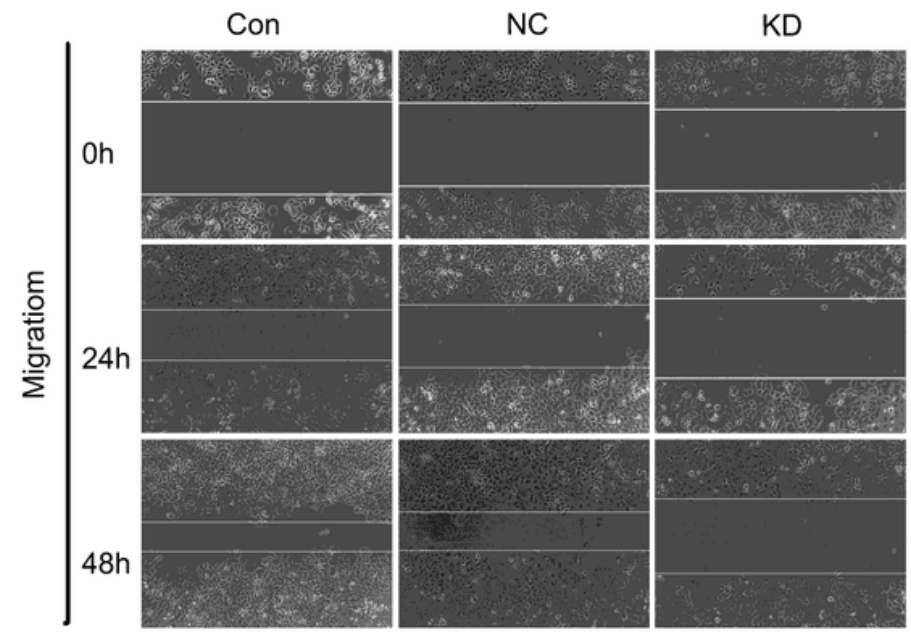

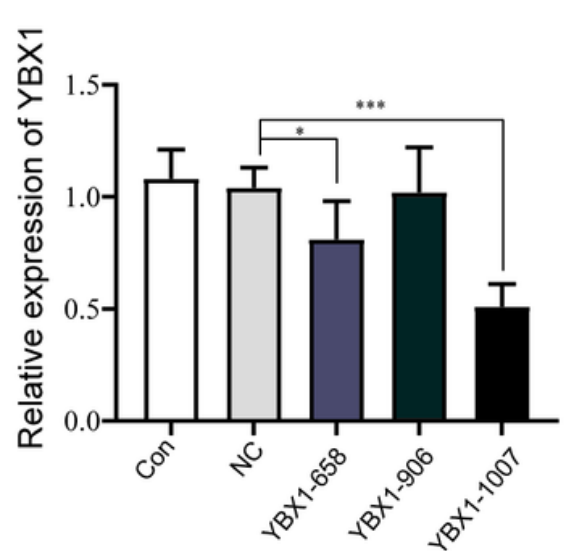

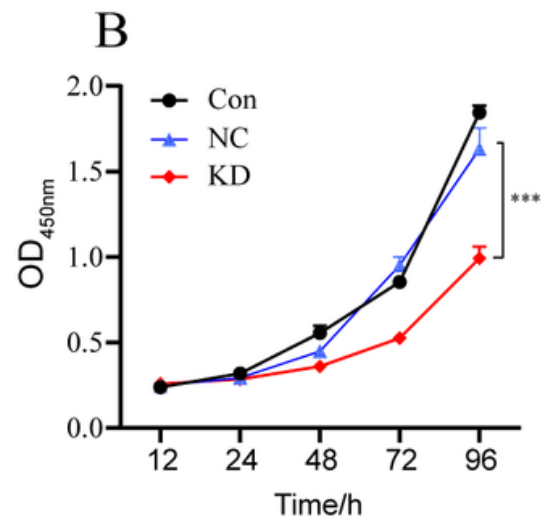

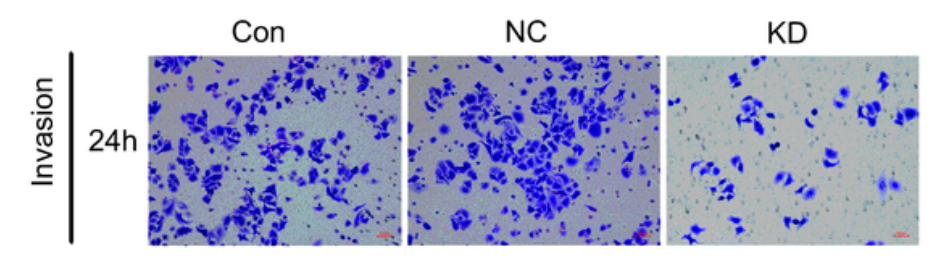
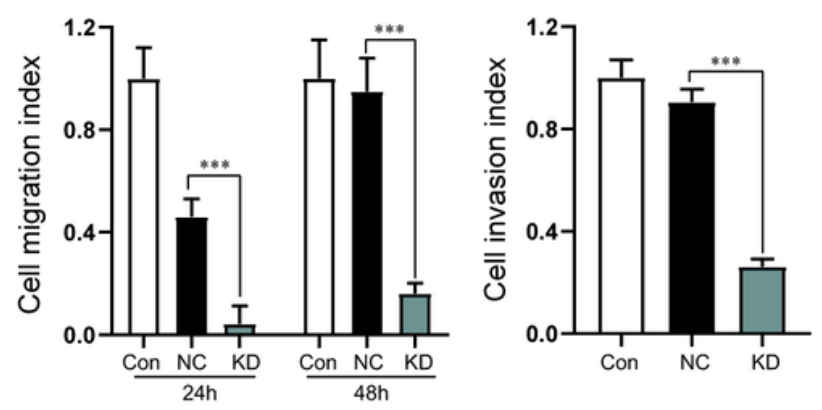

\section{Figure 2}

Transient YBX1 knockdown impairs LSCC cell in vitro migration, growth, and invasion. a Western blotting was used to confirm successful siRNA-mediated knockdown of YBX1 in Tu212 cells, with YBX1-1007 exhibiting optimal efficacy ( $n=3 /$ group; Student's t-tests). b Tu212 cell growth following siRNA transfection was assessed via MTT assay ( $n=6 /$ group; two-way ANOVA). c Wound healing and Transwell assays were used to assess cell migration and invasion, respectively, with migration and invasion indices being graphed ( $n=3 /$ group; Student's t-test). Data are means $\pm S D$. ${ }^{*} P<0.01,{ }^{*} * * 00.001$. 
A

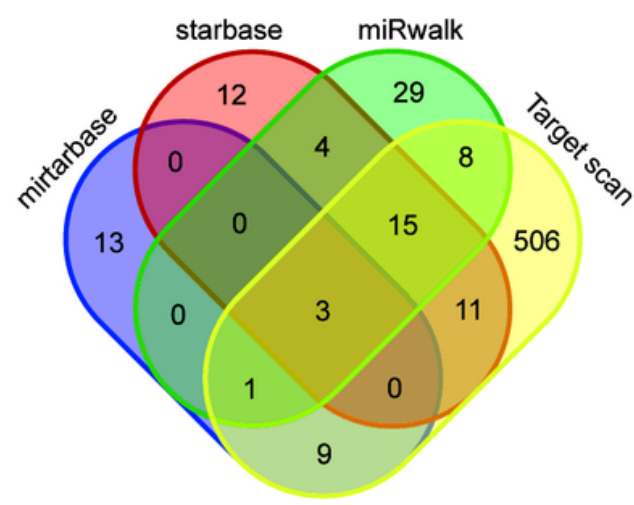

hsa-miR-379-5p hsa-miR-382-5p hsa-miR-137

\section{$\mathrm{D}$}

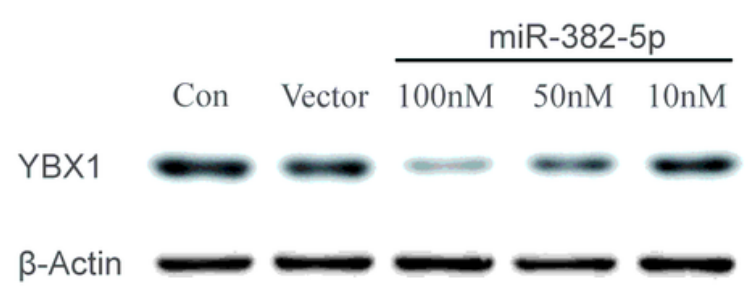

B

YBX1 WT 3'-UTR $\quad 5$ '...uguaauaaaaguuuACAACUUg... 3' miR-382-5p $\quad 3^{\prime}$...gcuuagguggugcuÚGUUGAAg... 5'

YBX1 Mutant 3'-UTR $\quad 5$ '...uguaauaaaaguuuGCATCUCg ... 3'

C
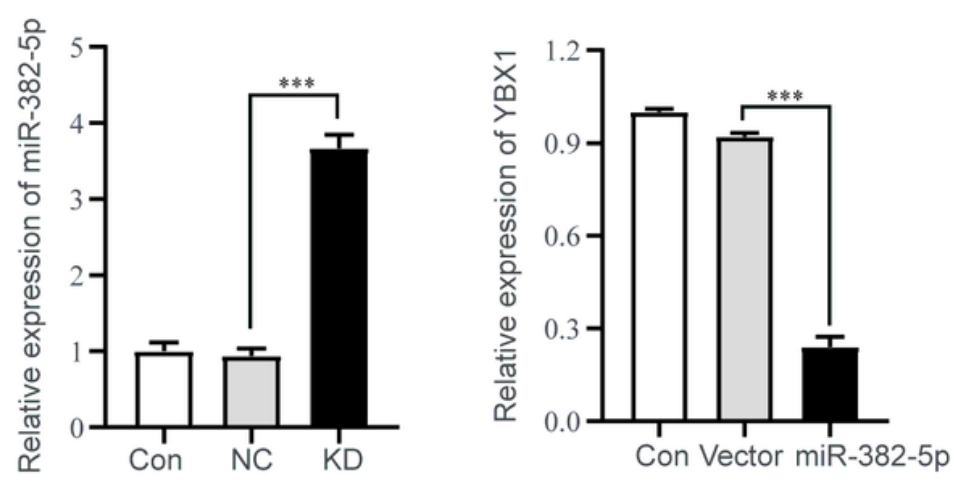

$\mathrm{E}$
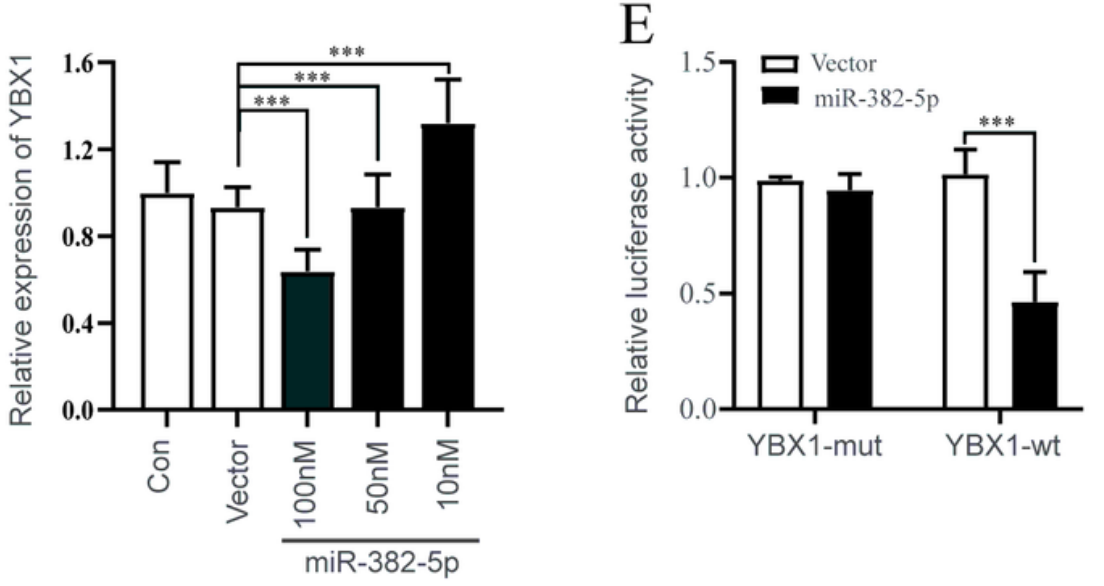

\section{Figure 3}

YBX1 is a miR-382-5p target in LSCC. a A schematic overview of overlapping putative miR-382-5p target genes in the miRtarbase, strabase, miRwalk, and Targetscan databases. b Identification of the putative overlap between miR-382-5p and the 3'-UTR of YBX1, with mutated residues in luciferase reporter constructs also being as indicated. c Transient YBX1 silencing enhanced miR-382-5p expression in Tu212 cells, while transfection with miR-382b-5p significantly suppressed YBX1 MRNA levels in these cells. $d$ YBX1 levels were assessed in Tu212 cells following transfection with a range of miR-382-5p concentrations $(10,50,100 \mu \mathrm{mol} / \mathrm{L})$. e Tu212 cells were transfected with pmirGLO YBX1-wt or pmirGLOYBX1-mt vectors together with miR-382-5p, after which luciferase activity was assessed. All data are means $\pm S D$. $* \star * P<0.001$; Student's t-tests. 
A
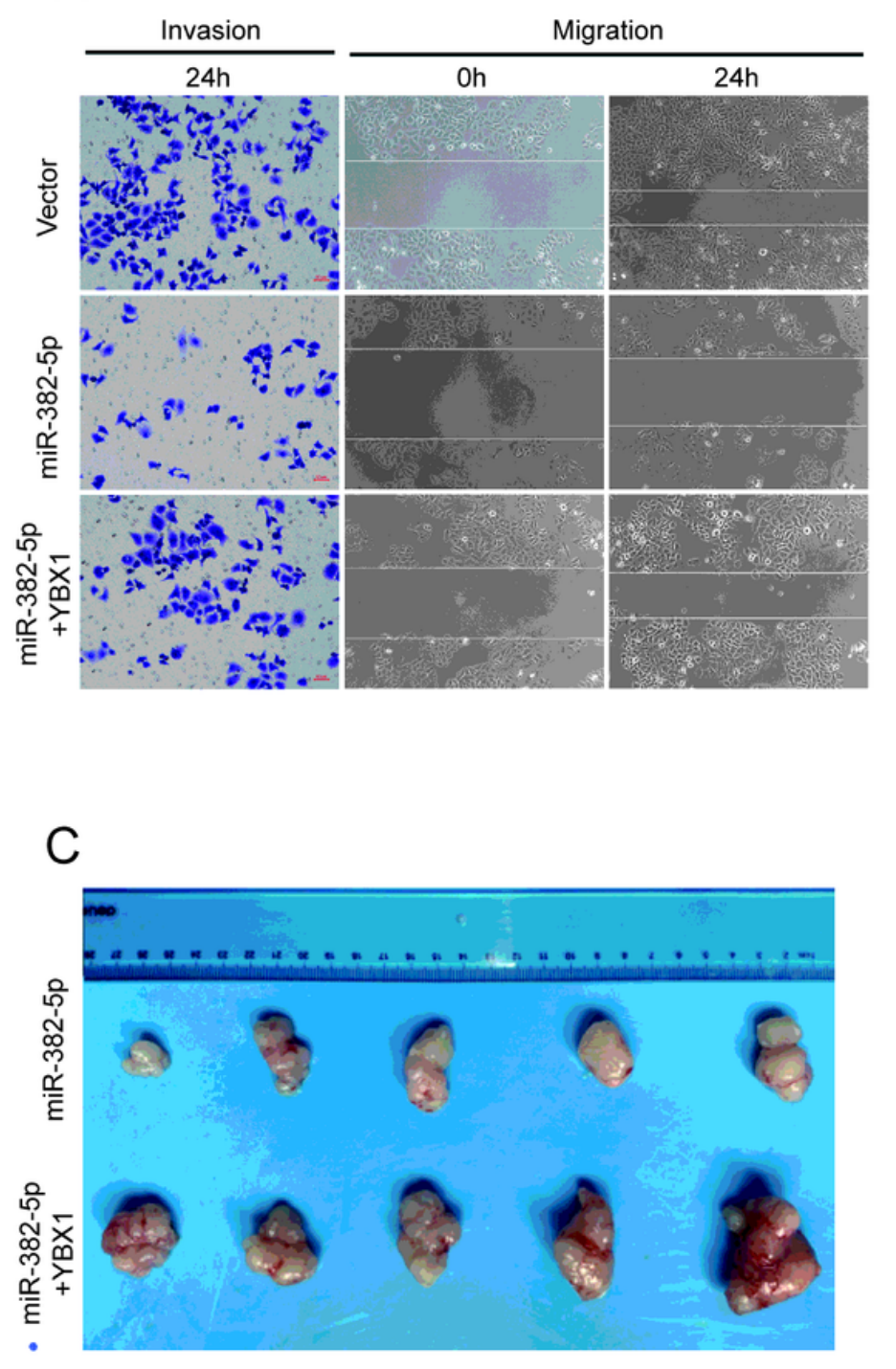
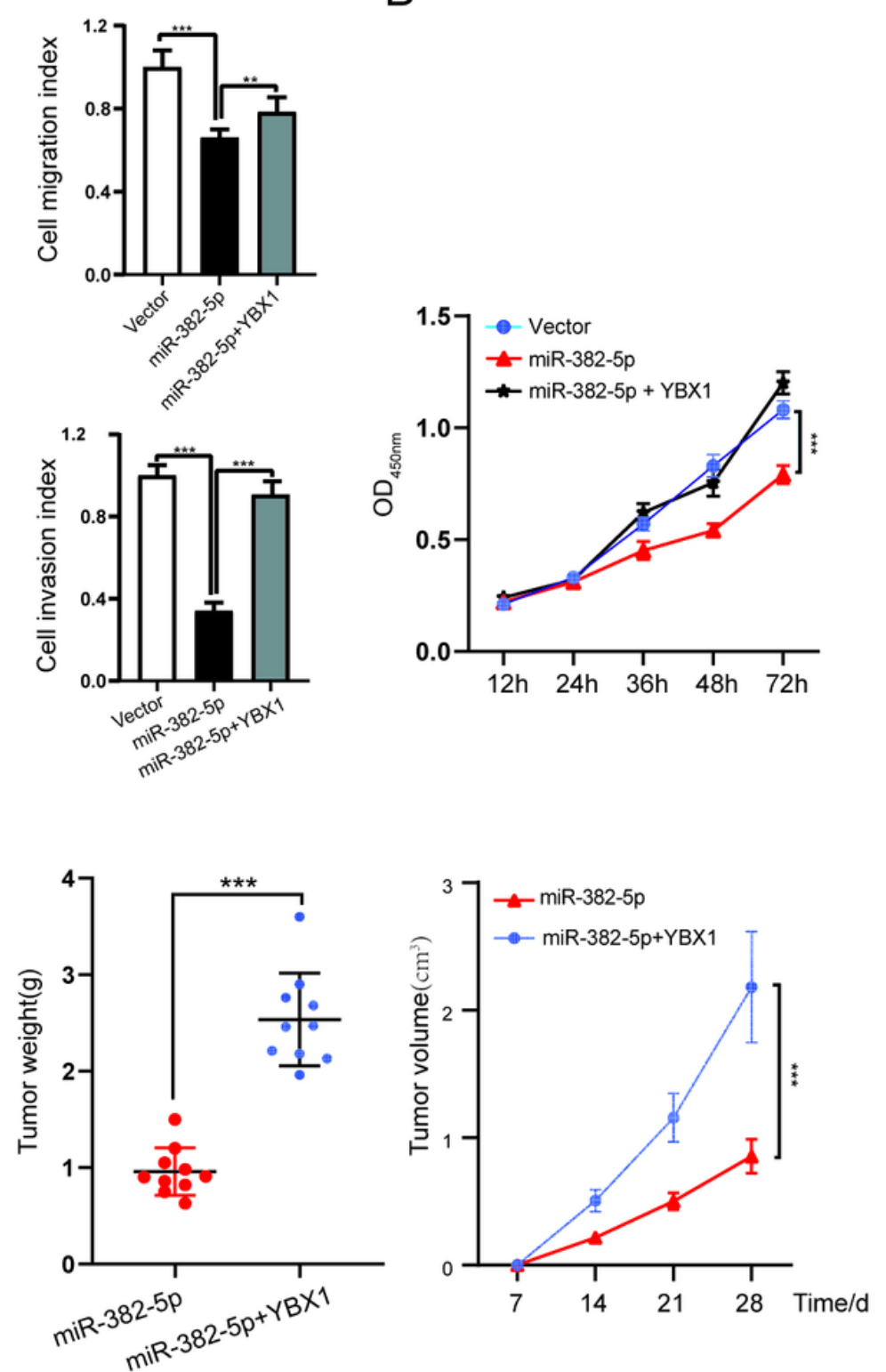

Figure 4

miR-382-5p inhibits the ability of YBX1 expression to inhibit LSCC growth. a The invasive and migratory activity of Tu212 cells was assessed via Transwell and wound healing assays, respectively, following miR-382-5p transfection and/or YBX1 overexpression ( $n=3$ /group). b An MTT assay was used to gauge the proliferation of Tu212 cells following miR-382-5p transfection and/or YBX1 overexpression ( $n=5 /$ group). $c$ The growth of Tu212 tumors in which miR-382-5p had or had not been expressed was assessed using a murine subcutaneous tumor implantation model ( $n=10 /$ group), with tumor weights and volumes being compared as indicated. Data are means $\pm S D$. ${ }^{\star *} P<0.01,{ }^{\star} * \star P<0.001$; Student's t-test. 
A
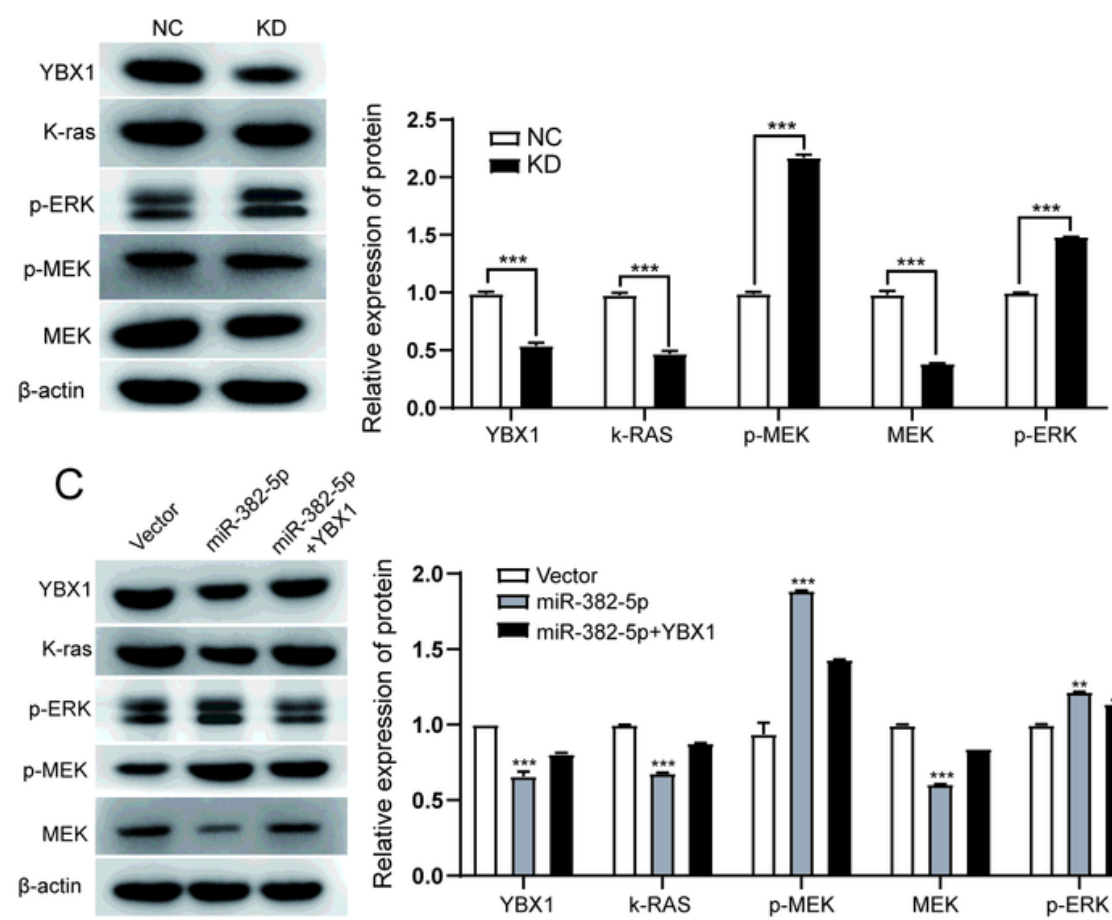

B

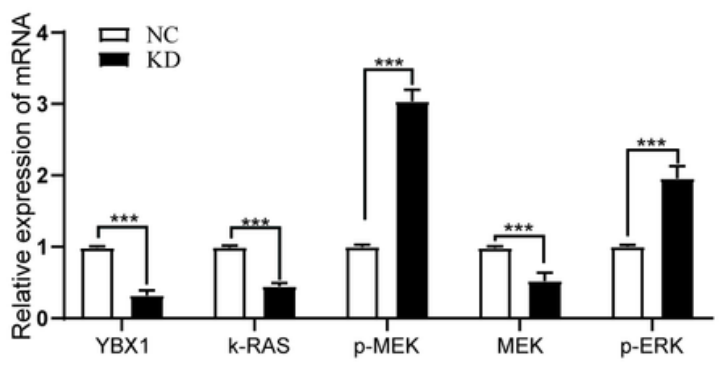

D

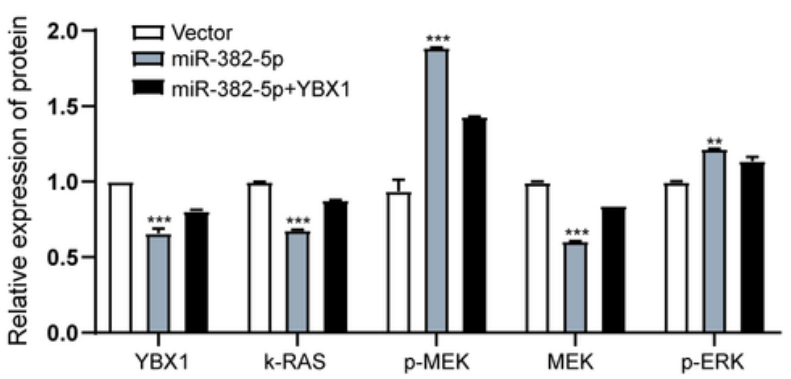

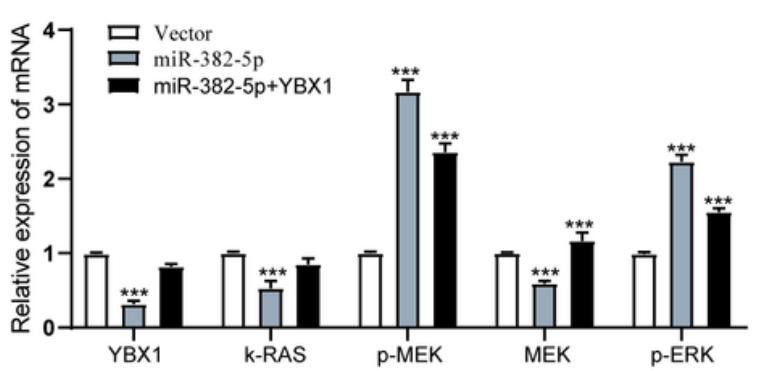

Figure 5

miR-382-5p inhibits the ability of YBX1 expression to drive LSCC growth via the MAPK/ERK signaling pathways. a MAPK/ERK signaling pathway components ( $k$-RAS, $p-M E K, p-E R K$, and MEK) were assessed in YBX1 knockdown or control cells via Western blotting b or by qPCR. c MAPK/ERK signaling pathway component expression was assessed in Tu212 cells transfected with miR-382-5p and/or overexpressing YBX1 via Western blotting $d$ or by $q P C R$. Data are means $\pm S D$. ${ }^{*} P<<0.01, * \star * P<0.001$; Student's t-test. 